\title{
Grau de desmineralização em osso trabecular e cortical por meio de tomografia computadorizada quantitativa em cães submetidos à terapia com prednisona
}

\author{
Trabecular and cortical bone demineralization degree by quantitative computed tomography \\ in dogs treated with prednisone
}

\author{
Lorena Adão Vescovi Séllos Costa ${ }^{I^{*}}$ Daniel Capucho de OliveiraI Juliana Giorgio Gianotti ${ }^{I}$ \\ Mauro Lahm Cardoso ${ }^{\text {II }}$ Fabiano Séllos Costa ${ }^{\text {III }}$
}

\section{RESUMO}

Os glicocorticóides são fármacos amplamente utilizados na medicina veterinária, entretanto, além dos efeitos benéficos, o seu emprego pode desencadear uma série de efeitos indesejados. Foi realizado um ensaio clínico em oito cães hígidos com o intuito de avaliar possiveis alterações na densidade mineral óssea após a terapia com prednisona, utilizando a tomografia computadorizada helicoidal. Todos os animais receberam a prednisona via oral na dose diária de $2 \mathrm{mg} \mathrm{kg}^{-1}$ de peso durante 30 dias. A densidade mineral óssea foi determinada a partir da obtenção de valores de radiodensidade da região de osso cortical e osso trabecular do corpo vertebral da segunda vértebra lombar, imediatamente antes e após o periodo de administração do medicamento. $O$ protocolo experimental permitiu a caracterização de significativa $(P<0,05)$ diminuição da radiodensidade do corpo vertebral da segunda vértebra lombar, entretanto não se constatou desmineralização significativa na região cortical. Nenhum dos animais apresentou fratura patológica ao término da administração do medicamento. O presente estudo comprovou que as alterações no metabolismo ósseo de cães submetidos à terapia com prednisona na dose de $2 \mathrm{mg} \mathrm{kg}^{-1}$ ocorrem precocemente somente em osso trabecular, tornandose recomendado um monitoramento dos pacientes para prevenção de fraturas patológicas.

Palavras-chave: cão, corticóide, densidade óssea, tomografia computadorizada.

\section{ABSTRACT}

Glucocorticoids are widely used in veterinary medicine. However, beyond the beneficial effects, their use can trigger a series of unwanted effects. A clinical trial was conducted in eight healthy dogs in order to assess possible changes in bone mineral density after therapy with prednisone using helical computed tomography. All animals received oral prednisone at a daily dose of $2 \mathrm{mg} \mathrm{kg}^{-1}$ of body weight for 30 days. Bone mineral density was determined by the obtaining of radiodensity values from the cortical region and the trabecular region of the second lumbar vertebra immediately before and after the period of drug administration. The experimental protocol allowed the characterization of a significant $(P<0.01)$ decrease of trabecular bone radiodensity on the second lumbar vertebra, but no significant demineralization was found in the cortical region. None of the dogs used in this experiment had pathological fracture at the end of the experimental protocol. This study showed that in dogs subjected to therapy with prednisone at a dose of $2 \mathrm{mg} \mathrm{kg}^{-1}$ changes in bone metabolism occur early in trabecular bone, being recommended to monitor these patients for the prevention of pathologic fractures.

Key words: dog, corticosteroids, bone mineral density, computed tomography.

\section{INTRODUÇÃO}

Os glicocorticóides representam um grupo de fármacos utilizado para o tratamento de diversos sinais clínicos e enfermidades pela sua ação antiinflamatória, imunossupressora (MACDONALD, 2004; COHN, 2006), como parte do tratamento antineoplásico em protocolos quimioterápicos e no tratamento de síndromes paraneoplásicas $(\mathrm{COHN}$, 2006). Apesar dos efeitos benéficos, quadros de

\footnotetext{
'Departamento de Medicina Veterinária, Centro de Ciências Agrárias (CCA), Universidade Federal do Espírito Santo (UFES), Alto Universitário, s/n, CP 16, 29500-000, Alegre, ES, Brasil. E-mail: lovescovi@gmail.com. *Autor para correspondência.

"Universidade Estadual do Norte do Paraná (UENP), campus Luiz Meneghel (CLM), Bandeirantes, PR, Brasil.

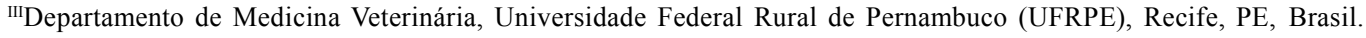


toxicidade e alterações metabólicas podem ocasionar importantes efeitos colaterais (BADYLAK \& VAN FLEET, 1981; SCHÄCKE et al., 2002; MACDONALD, 2004; COHN, 2006).

Os efeitos do uso de corticóides sobre o metabolismo ósseo são pouco descritos em animais domésticos, sendo os trabalhos realizados direcionados principalmente para a utilização de modelos animais para estudos experimentais (NORDIN et al., 1990; QUARTES, 1992; LYLES et al., 1993; LILL et al., 2002; ENGERMANN et al., 2005; SCHOLZ-AHRENS et al., 2007). Em humanos, estudos revelam que tanto a corticoterapia prolongada (GRAMPP et al., 1996; ADACHI et al., 1997; BELL et al., 1997; LANE \& LUKERT, 1998; PEARCE et al., 1998; GOLDSTEIN et al., 1999; MONOLAGAS \& WEINSTEIN, 1999; REID, 2000; ETTINGER et al., 2001; SCHÄCKE et al., 2002; KANIS et al., 2007; DE NIJS, 2008) como a ocorrência de Síndrome de Cushing (MANCINI et al., 2004; VAN DER EERDEN et al., 2007; FUTO et al., 2008) de origem endógena podem ocasionar osteopenia e osteoporose com consequente aumento na ocorrência de fraturas patológicas. Embora os mecanismos desses eventos sejam complexos, múltiplos fatores podem estar envolvidos, promovendo inibição da atividade osteoblástica e aumento da atividade osteoclástica (KANIS et al.,2007; DE NIJS, 2008).

A prednisona é um glicocorticóide sintético de ação intermediária que apresenta grande potência antiinflamatória. A prednisona é biologicamente inerte, sendo convertida no fígado em sua forma ativa, a prednisolona (MACDONALD, 2004; COHN, 2006). Estudos realizados em cães submetidos à corticoterapia (BADYLAK \& VANFLEET, 1981; RUTGERS etal., 1995; O'BRIEN et al., 1996; LU et al., 1997; SYAKALIMA et al., 1998; MANCINI et al., 2004) descrevem inúmeras alterações clínicas e laboratoriais. Nosso objetivo foi avaliar comparativamente, por meio da tomografia computadorizada (TC), o grau de desmineralização na região de osso cortical e osso trabecular de cães, submetidos à terapia com prednisona.

\section{MATERIAL E MÉTODOS}

Para a seleção do grupo experimental foram realizados exames de triagem que envolveram uma acurada avaliação clínica, incluindo exame físico completo, assim como realização de hemograma, urinálise e dosagem dos níveis séricos de ALT, AST, FA, GGT, uréia, creatinina, proteína total, albumina, cortisol, T4 livre e T4 total. O grupo experimental foi composto de oito cães adulto-jovens, hígidos, não castrados, sem distinção de raça ou sexo e com peso corporal oscilando entre 10 e $15 \mathrm{~kg}$. Os cães foram alojados em canis individuais com acesso à luz solar, recebendo água e ração do tipo premium (Purina ${ }^{\circledR}$ ProPlan $^{\circledR}$ ) à vontade.

Os exames de TC foram realizados em dois momentos. O momento inicial (M0) antes da administração de prednisona correspondeu ao primeiro dia do experimento. Depois do exame tomográfico, iniciou-se a administração de prednisona na dose de $2 \mathrm{mg} \mathrm{kg}^{-1}$ por via oral a cada 24 horas (M1). A medicação foi ministrada durante 30 dias e foi o $31^{\circ}$ dia que correspondeu ao momento final do protocolo experimental (M2), quando se realizaram os exames tomográficos. Os animais eram semanalmente pesados e as doses ministradas de prednisona eram reajustadas de acordo com o peso do animal. Depois do M2 (ao término do experimento), realizou-se uma gradativa diminuição das doses do fármaco durante três semanas, sendo os animais posteriormente doados.

Para a realização dos exames de $\mathrm{TC}$, os animais foram submetidos a jejum hídrico e alimentar. Instituiu-se de fluidoterapia com solução fisiológica em velocidade de infusão de $10 \mathrm{ml} \mathrm{kg}^{-1} \mathrm{~h}^{-1}$ e prémedicação e indução anestésica com diazepam e propofol nas doses de $0,5 \mathrm{mg} \mathrm{kg}^{-1}$ de peso e $6 \mathrm{mg} \mathrm{kg}^{-1} \mathrm{de}$ peso, respectivamente. Os animais foram posicionados em decúbito lateral direito, sendo realizados os exames de TC com o aparelho GE Hi-Speed FXI e protocolo com $120 \mathrm{kVp}$ e auto $\mathrm{mA}$ na velocidade de uma rotação por segundo. A aquisição das imagens foi obtida em cortes transversais de $2 \mathrm{~mm}$ de espessura com filtro para partes ósseas. Antecedendo os exames, o aparelho foi calibrado para melhor padronização dos resultados.

Após o exame tomográfico e digitalização das imagens, realizou-se a identificação do corpo vertebral da segunda vértebra lombar em cada animal. A estimativa da radiodensidade do tecido ósseo foi realizada após obtenção do valor médio de três regiões de interesse (ROI), selecionadas do osso trabecular do corpo vertebral e osso cortical (Figura 1). Para padronização dos níveis de corte e do local de obtenção das medidas de radiodensidade do tecido ósseo, levouse em consideração a visualização similar dos processos espinhosos e transversos da segunda vértebra lombar.

A análise estatística das variáveis foi realizada por meio do Teste t para estudos pareados, com o nível de significância 0,05 .

\section{RESULTADO E DISCUSSÃO}

Os resultados demonstraram uma significativa diminuição da densidade mineral óssea 


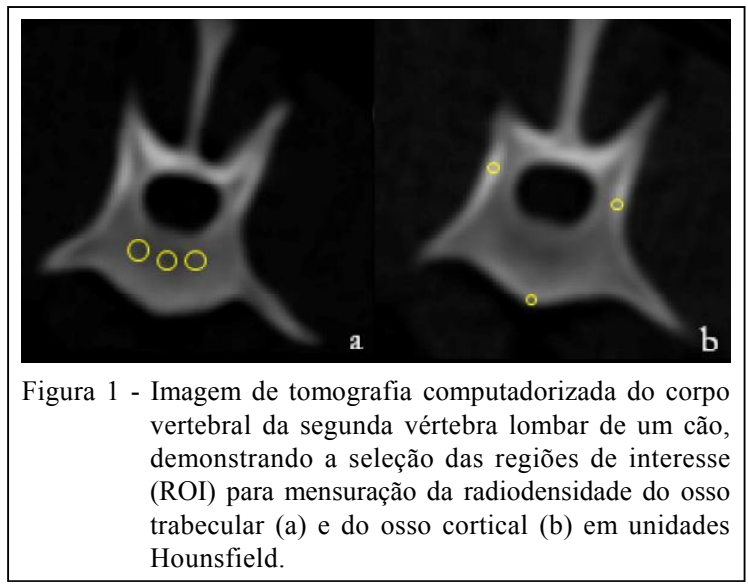

do osso trabecular do corpo vertebral da segunda vértebra lombar entre os momentos M1 e M2 do experimento. Entretanto, não se verificou diferença estatística significativa entre os momentos após a mensuração da radiodensidade em osso cortical. Em nenhum animal foi diagnosticada a ocorrência de fratura patológica, mas os valores médios de radiodensidade óssea demonstraram uma perda de massa óssea de aproximadamente $17 \%$ em osso trabecular. Os valores médios das densidades nas regiões cortical e trabecular do corpo vertebral da segunda vértebra lombar encontram-se descritos respectivamente nas tabelas 1 e 2 .

Apesar de os efeitos colaterais da terapia com glicocorticóides serem considerados bem comprovados em cães e seus efeitos adversos serem considerados maiores no homem do que em animais, a literatura pouco aborda sobre a intensidade dos efeitos deletérios sobre o metabolismo ósseo nesta espécie (NORDIN et al., 1990; QUARTES, 1992; LYLES et al., 1993). Em humanos, inúmeras pesquisas procuram aumentar o conhecimento sobre diversos aspectos epidemiológicos e fisiopatológicos dos efeitos

Tabela 1 - Valores médios e desvio padrão de radiodensidade em unidades Hounsfield do osso trabecular do corpo vertebral da segunda vértebra lombar, em cães submetidos à terapia com prednisona por 30 dias.

\begin{tabular}{lcc}
\hline DMO & M1 & M2 \\
\hline ROI 1 & $453 \pm 71 \mathrm{HU}^{\mathrm{b}}$ & $388 \pm 75 \mathrm{HU}^{\mathrm{a}}$ \\
ROI 2 & $375 \pm 71 \mathrm{HU}^{\mathrm{b}}$ & $331 \pm 48 \mathrm{HU}^{\mathrm{a}}$ \\
ROI 3 & $438 \pm 80 \mathrm{HU}^{\mathrm{b}}$ & $375 \pm 61 \mathrm{HU}^{\mathrm{a}}$ \\
Média & $425 \pm 69 \mathrm{HU}^{\mathrm{b}}$ & $365 \pm 59 \mathrm{HU}^{\mathrm{a}}$ \\
\hline
\end{tabular}

M0: momento inicial; M1: momento final; DMO: densidade mineral óssea; ROI: região de interesse; HU: unidades Hounsfield. Letras diferentes na mesma linha diferem pelo teste $T$ pareado em nível de 5\% de significância.
Tabela 2 - Valores médios e desvio padrão de radiodensidade em unidades Hounsfield do osso cortical do corpo vertebral da segunda vértebra lombar, em cães submetidos à terapia com prednisona por 30 dias.

\begin{tabular}{lll}
\hline DMO & \multicolumn{1}{c}{ M1 } & \multicolumn{1}{c}{ M2 } \\
\hline ROI 1 & $844 \pm 175 \mathrm{HU}^{\mathrm{a}}$ & $868 \pm 186 \mathrm{HU}^{\mathrm{a}}$ \\
ROI 2 & $870 \pm 169 \mathrm{HU}^{\mathrm{a}}$ & $810 \pm 90 \mathrm{HU}^{\mathrm{a}}$ \\
ROI 3 & $685 \pm 33 \mathrm{HU}^{\mathrm{a}}$ & $669 \pm 93 \mathrm{HU}^{\mathrm{a}}$ \\
Média & $799 \pm 100 \mathrm{HU}^{\mathrm{a}}$ & $783 \pm 102 \mathrm{HU}^{\mathrm{a}}$ \\
\hline
\end{tabular}

M0: momento inicial; M1: momento final; DMO: densidade mineral óssea; ROI: região de interesse; HU: unidades Hounsfield. Letras diferentes na mesma linha diferem pelo teste $\mathrm{T}$ pareado em nível de 5\% de significância.

colaterais deste grupo de fármacos sobre o tecido ósseo (GRAMPP et al., 1996; ADACHI et al., 1997; BELL et al., 1997; LANE \& LUKERT, 1998; PEARCE et al., 1998; GOLDSTEIN et al., 1999; MONOLAGAS \& WEINSTEIN, 1999; REID, 2000; ETTINGER etal., 2001; SCHÄCKE et al., 2002; KANIS et al., 2007; DE NIJS, 2008).

A osteoporose induzida pela corticoterapia é um dos mais importantes problemas mundiais de saúde em humanos, fato agravado pelo aumento da expectativa de vida da população (KANIS et al., 2007). Os corticóides são capazes de gerar alterações sistêmicas caracterizada por perda de massa óssea e deterioração da microarquitetura do tecido ósseo, promovendo aumento de fragilidade e susceptibilidade a fraturas (SCHÄCKE et al., 2002; KANIS et al., 2007). Apesar dessa situação, estudos demonstram que aproximadamente $50 \%$ dos pacientes que recebem altas doses de corticóides não são investigados para avaliação de um possível quadro de osteoporose (BELL et al., 1997) e apenas $10 \%$ recebem tratamento preventivo com suplementação de cálcio (ETTINGER et al., 2001; SCHÄCKE et al., 2002). A proximadamente $33 \%$ dos pacientes humanos que fazem uso contínuo de corticóides apresentam fraturas patológicas compressivas em vértebras após um período de 5 a 10 anos de terapia, sendo esse fato particularmente mais preocupante em mulheres após a menopausa (ADACHI et al., 1997).

A dose de prednisona administrada no presente estudo é a mesma utilizada na rotina de atendimento clínico de cães no tratamento de dermatopatias, doenças articulares e doenças neurológicas. Esta dosagem também foi utilizada por outros autores (O'BRIEN et al., 1996; LU et al., 1997), permitindo a caracterização de alterações precoces ao exame ultrassonográfico, decorrentes de hepatopatia esteroidal induzida, tais como hepatomegalia e aumento 
difuso de ecogenicidade do parênquima. Outros estudos também avaliaram os efeitos colaterais do uso de corticóides em cães, caracterizando diversas alterações clínicas e laboratoriais (BADYLAK \& VAN FLEET, 1981; NORDIN et al., 1990; QUARTES, 1992; LYLES et al., 1993; RUTGERS et al., 1995).

A análise quantitativa da densidade mineral óssea por TC permitiu a determinação da radiodensidade do osso trabecular e cortical nos cães do grupo experimental. A TC é uma técnica com alta sensibilidade, aplicabilidade e precisão no diagnóstico de perda de massa óssea, assim como para a identificação de fraturas vertebrais decorrentes de osteoporose (GRAMPP et al., 1996). Comparando-se com a absorção de raios-X de dupla energia (DEXA), a TC permite quantificar isoladamente as regiões de osso cortical e osso trabecular, enquanto que a DEXA fornece informações da densidade óssea como um todo, não permitindo uma avaliação individualizada das regiões de osso cortical e trabecular (ADAMS, 2009). Estudos experimentais utilizaram com sucesso a TC para avaliação da densidade mineral óssea da coluna lombar em cães, ovelhas e minipigs (QUARTES, 1992; LYLES et al., 1993; AERSSENS et al., 1998; LILL et al., 2002; SCHOLZ-AHRENS et al., 2007).

A forma que os glicocorticóides induzem a osteoporose é considerada complexa e ainda não completamente elucidada. $\mathrm{O}$ maior efeito sobre o metabolismo ósseo em humanos é um decréscimo da formação óssea associada a uma reabsorção óssea diminuída ou inalterada. Os glicocorticóides afetam a diferenciação, a atividade e o tempo de vida média dos osteoblastos e osteócitos, diminuindo consequentemente a formação óssea. Os fatores que promovem o aumento da reabsorção óssea não são claros, mas podem envolver um prejuízo na absorção intestinal de cálcio e uma deficiência de hormônios sexuais (SCHÄCKE et al., 2002; KANIS et al., 2007).

A carência de trabalhos utilizando modelos animais para estudar os efeitos dos glicocorticóides sobre o metabolismo ósseo é considerada um dos motivos da não elucidação de muitos aspectos envolvidos na fisiopatologia da osteoporose induzida pelo medicamento (ENGERMANN et al., 2005). Estudos em ovelhas após administração de esteróides demonstram alterações estruturais sobre o tecido ósseo dos corpos vertebrais e fêmur, verificando-se que as alterações, de acordo com o grau de osteoporose, são comparáveis com as observadas em humanos (LILL et al., 2002).

A literatura relata amplamente que o aparecimento dos efeitos adversos sobre o tecido ósseo é observado após terapia prolongada por glicocorticóides em humanos (GRAMPP et al., 1996; ADACHI et al., 1997; BELL et al., 1997; LANE \& LUKERT, 1998; PEARCE et al., 1998; GOLDSTEIN et al., 1999; MONOLAGAS \& WEINSTEIN, 1999; REID, 2000; ETTINGER et al., 2001; SCHÄCKE et al., 2002; KANIS et al., 2007; DE NIJS, 2008). Os resultados do presente estudo comprovaram uma diminuição significativa da densidade mineral óssea com um mês de administração do fármaco, fato que ocorreu em todos os animais do grupo experimental. É citado que humanos apresentam uma perda de massa óssea aproximada de $12 \%$ após alguns meses de terapia com corticóides (MONOLAGAS \& WEINSTEIN, 1999), entretanto, o protocolo empregado demonstrou em apenas 30 dias uma desmineralização de cerca de 17\%, portanto, um grau superior ao descrito nesta espécie. Dessa forma, não podemos considerar que os efeitos deletérios da corticoterapia em animais, particularmente sobre o metabolismo mineral ósseo, sejam menos significativos quando comparados com humanos.

Durante avaliação densitométrica ao exame tomográfico do osso cortical, as superfícies endosteal e periosteal podem causar o artefato de efeito de volume parcial em regiões de osso cortical com espessura inferior a de $2 \mathrm{~mm}$ (HANGARTNER \& GILSANZ, 1996). Assim, deve-se considerar a presença desse artefato para a interpretação dos valores densitométricos, principalmente na avaliação de regiões de osso cortical com espessura menor que $2 \mathrm{~mm}$, fato que não ocorreu neste experimento.

Assim como em humanos, caracterizou-se uma rápida desmineralização em osso trabecular dos cães decorrentes da corticoterapia, enquanto que o osso cortical não apresentou variações estatisticamente significativas. Segundo ADAMS (2009), isso se justifica pelo fato de que o osso trabecular é metabolicamente mais ativo quando comparado ao osso cortical, apresentando, dessa forma, maior suscetibilidade à ação de fármacos do que alterações ao metabolismo mineral ósseo. Deve-se considerar a possibilidade de desmineralização em osso cortical em terapias de longo prazo, aumentado a possibilidade de fraturas patológicas nos pacientes.

\section{CONCLUSÃO}

Conclui-se que o uso de prednisona na dose diária de $2 \mathrm{mg} \mathrm{kg}^{-1}$ de peso é capaz de promover desmineralização precoce do osso trabecular em cães, o mesmo não foi observado na região cortical. A tomografia computadorizada mostrou-se eficiente em quantificar a desmineralização no osso trabecular. 
Outros estudos tornam-se necessários para avaliar os possíveis efeitos de protocolos de corticoterapia diferentes, estudar a eficácia de medidas terapêuticas preventivas para osteoporose, bem como investigar a razão da precocidade de resposta em cães, quando comparados com a resposta em humanos.

\section{COMITÊ DE ÉTICA E BIOSSEGURANÇA}

Este estudo foi realizado sob aprovação do Comitê de Ética e Bem-estar Animal da Universidade Federal do Espírito Santo (UFES), tendo sido respeitados todos os preceitos éticos de proteção aos animais.

\section{REFERÊNCIAS}

ADACHI, J. et al. Intermittent etidronato therapy to prevent corticosteroid-induced osteoporosis. New England Journal of Medicine, v.337, p.382-387, 1997.

ADAMS, J.E. Quantitative computed tomography. European Journal of Radiology, v.71, p.415-424, 2009. Disponível em: <http://www.ncbi.nlm.nih.gov/pubmed/19682815>. Acesso em: 14 out. 2010. doi:10.1016/j.ejrad.2009.04.074.

AERSSENS, J. et al. Interspecies differences in bone composition, density, and quality: potential implications for in vivo bone research. Endocrinology, v.139, p.663-670, 1998. Disponível em: <http://endo.endojournals.org/cgi/ search? fulltext $=$ Interspecies + differences + in + bone + compositio $\mathrm{n} \% 2 \mathrm{C}+$ density $\% 2 \mathrm{C}+$ and + quality $\% 3 \mathrm{~A}+$ potential+implications + for + in + vi\&volume $=139 \&$ issue $=2 \&$ journalcode $=$ endo $>$. Acesso em: 14 out. 2010 . doi: 10.1210/en.139.2.663.

BADYLAK, S.; VAN FLEET, J. Sequential morphologic and clinicopathologic alterations in dogs with experimentally induced glucocorticoide hepatopathy. American Journal of Veterinary Research, v.42, p.1310-1318, 1981.

BELL, R. et al. Managing corticosteroid-induced osteoporosis in medical outpatientes. Journal of Rowall College Physicians London, v.31, p.158-161, 1997.

COHN, L.A. Glucocorticoid therapy. In: ETTINGER SJ, F.E. (Ed.). Textbook of veterinary internal medicine. St. Louis: Elsevier Saunders, 2006. p.503-508.

DE NIJS, R. Glucocorticoid-induced osteoporosis: a review on pathophysiology and treatment options. Minerva Medica, v.99, p.23-43, 2008.

ENGERMANN, M. et al. Animal models for fracture treatment in osteoporosis. Osteoporosis International, v.16, p.129138, 2005. Disponível em: <http://link.periodicos.capes.gov.br/

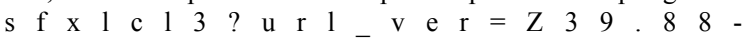
2004\&url_ctx_fmt $=$ infofifmt:kev:mtx:ctx\&ctx_enc=info:ofi/ enc:UTF-8\&ctx_ver=Z39.88-2004\&rfr_id =info:sid/ sfxit.com:azlist\&sfx.ignore_date_threshold=1\&rft.object_ $\overline{\mathrm{id}}=95492557187>$. Acesso em:14 out. 2010 . doi 10.1007/s00198-005-1859-7.

ETTINGER, B. et al. Prevalence and determinants of osteoporosis drug prescription among patients with high exposure to glucocorticoid drugs. American Journal of Managed Care, v.7, p.597-605, 2001. Disponível em: < http://www.ajmc.com/
media/pdf/AJMC2001junEttinger597_605.pdf>. Acesso em: 14 out.2010.

FÜTO, L. et al. Skeletal differences in bone mineral area and content before and after cure of endogenous Cushing's syndrome. Osteoporosis International, v.19, p.941-949, 2008. Disponível em: <http://www.springerlink.com/content/ 0140376u74562mp4/fulltext.pdf>. Acesso em: 14 out.2010. doi: $10.1007 / \mathrm{s} 00198-007-0514-x$.

GOLDSTEIN, M. et al. Chronic glucocorticoid therapyinduced osteoporosis in patients with obstructive lung disease. Chest, v.116, p.1733-1749, 1999. Disponível em: <http:// chestjournal.chestpubs.org/content/116/6/ 1733.full.pdf + html $>$. Acesso em: 14 out. 2010. doi: 10.1378/ chest.116.6.1733.

GRAMPP, S. et al. Quantitative CT assessment of the lumbar spine and radius in patients with osteoporosis. AJR American Journal of Roentgenology, v.167, p.133-140, 1996. Disponível em: <http://www.ajronline.org/cgi/reprint/167/1/ 133 ? maxtoshow $=\&$ hits $=10 \&$ RESULTFORMAT $=\&$ fulltext $=\mathrm{Q}$ uantitative $+\mathrm{CT}+$ assessment + of + the + lumbar + spine + and + radius + in + patients + with + osteoporosis \&searchid $=1$ \&FIRSTINDEX $=0$ \& volume $=167 \&$ issue $=1 \&$ resourcetype $=$ HWCIT $>$. Acesso em: 14 out. 2010 .

HANGARTNER, T.V.; GILSANZ, V. Evaluation of cortical bone by computed tomography. Journal of Bone and Mineral Research, v.11, n.10, p.1518-1525, 1996. Disponível em: $<$ http://www.ncbi.nlm.nih.gov/pubmed/8889852>. Acesso em: 14 out. 2010.

KANIS, J. et al. Glucocorticoid-induced osteoporosis: a systematic review and cost-utility analysis. Health Technology Assessment, v.11, n.7, p.iii-iv, ix-xi, 1-231, 2007. Disponível em : <http://www.hta.ac.uk/pdfexecs/summ1107.pdf>. Acesso em: 14 out. 2010.

LANE, N.; LUKERT, B. The science and therapy of GCinduced bone loss. Metabolism Clinics of North America, v.27, p.465-483, 1998.

LILL, C. et al. Bone changes due to glucocorticoid application in an ovariectomized animal model for fracture treatment in osteoporosis. Osteoporos International, v.13, p.407-414, 2002. Disponível em: <http://www.ncbi.nlm.nih.gov/pubmed/ 12086352>. Acesso em: 14 out. 2010.

LU, Z. et al. Ultrasound attenuation and backscatter in the liver during prednisone administration. Ultrasound in Medicine and Biology, v.23, p.1-8, 1997. Disponível em: $<$ http://www.umbjournal.org/article/S0301-5629(96)00181-0/ abstract>. Acesso em: 15 out. 2010.

LYLES, K. et al. Salmon calcitonin reduces vertebral bone loss in glucocorticoid-treated beagles. American Journal of Physiology Endocrinology Metabolism, v.264, p.938-942, 1993.

MACDONALD, J.M. Corticoterapia. In: ETTINGER S.J. (Ed.). Tratado de medicina interna veterinária. Rio de Janeiro: Guanabara Koogan, 2004. p.323-333.

MANCINI, T. et al. Cushing syndrome and bone. Pituitary, v.7, p.249-252, 2004. Disponível em: <http://www.ingentaconnect.com/ 
content/klu/pitu/2004/00000007/00000004/00001051>. Acesso em: 14 out. 2010 . doi: 10.1007/s11102-005-1051-2.

MONOLAGAS, S.; WEINSTEIN, R. New developments in the pathogenesis and treatment of steroid induced osteoporosis. Journal of Bone and Mineral Research, v.14, p.10611066, 1999. Disponível em: <http://onlinelibrary.wiley.com/ doi/10.1359/jbmr.1999.14.7.1061/full>. Acesso em: 14 out. 2010. doi: 10.1359/jbmr.1999.14.7.1061.

NORDIN, R. et al. Effects of corticosteroids on mechanical strength of intervertebral joints and vertebrae in dogs. Clinical Orthopaedics and Related Research , v.259, p.268-276, 1990.

O'BRIEN, R. et al. Measurement of acoustic backscatter and attenuation in the liver of dogs with experimentally induced steroid hepatopathy. American Journal of Veterinary Research, v.57, p.1690-1694, 1996.

PEARCE, G. et al. The deleterious effects of low-dose corticosteroids on bone density in patients with polymyalgia rheumatic. British Journal of Rheumatology, v.37, p.292299, 1998.

QUARTES, L. Prednisone induced osteopenia in beagles: variable effects mediated by differential suppression of bone formation. Journal of Physiology Endocrinology Metabolism, v.263, p.136-141, 1992.
REID, I. Glucocorticoid-induced osteoporosis. Baillière's Clinical Endocrinology and Metabolism , v.14, p.279298, 2000.

RUTGERS, C. et al. Subcellular pathologic features of glucocorticoid-induced hepatopathy in dogs. American Journal of Veterinary Research, v.56, p.898-907, 1995.

SCHOLZ-AHRENS, K. et al. Glucocorticosteroid-induced osteoporosis in adult primaparous Gottingen miniature pigs: effects on bone mineral and mineral metabolism. Journal of Physiology Endocrinology Metabolism, v.293, p.385-395, 2007.

SCHÄCKE, H. et al. Mechanisms involved in the side effects of glucocorticoids. Pharmacology and Therapeutics, v.96, p.23-43, 2002.

SYAKALIMA, M. et al. Comparison attenuation and liverkidney contrast of liver ultrasonographs with histology and biochemistry in dogs with experimentally induced steroid hepatopathy. Veterinary Quarterly, v.20, p.18-22, 1998.

VAN DER EERDEN, A. et al. Cushing syndrome and bone mineral density: lowest $Z$ scores in young patients. Netherlands Journal of Medicine , v.65, p.137-141, 2007. Disponível em: $<$ http://www.njmonline.nl/njm/getpdf.php?t=a\&id=10000167>. Acesso 14 out. 2010. 